\title{
Determinação do volume de madeira em povoamento de eucalipto por escâner a laser aerotransportado
}

\author{
Luciano Teixeira de Oliveira(1), Maria Zélia Ferreira ${ }^{(2)}$, Luis Marcelo Tavares de Carvalho(1), \\ Antônio Carlos Ferraz Filho(1), Thomaz Chaves de Andrade Oliveira ${ }^{(3)}$, \\ Eduarda Martiniano de Oliveira Silveira ${ }^{(1)}$ e Fausto Weimar Acerbi Junior ${ }^{(1)}$
}

\begin{abstract}
(1)Universidade Federal de Lavras, Departamento de Ciências Florestais, CEP 37200-000 Lavras, MG, Brasil. E-mail: oliveiralt@hotmail.com, passarinho@dcf.ufla.br, antoniocarlos.ferraz@dcf.ufla.br, dudalavras@hotmail.com, fausto@dcf.ufla.br (2)Veracel Celulose, Caixa Postal 21, CEP 45820-970 Eunápolis, BA, Brasil. E-mail: maria.zelia@veracel.com ${ }^{(3)}$ Universidade Federal de Lavras, Departamento de Ciências da Computação, CEP 37200-000 Lavras, MG, Brasil. E-mail: thomazchaves@gmail.com
\end{abstract}

Resumo - O objetivo deste trabalho foi avaliar a possibilidade de se estimar o diâmetro à altura do peito (DAP) com os dados de altura e de número de árvores derivados do escâner a laser aerotransportado (LiDAR, "light detection and ranging"), e determinar o volume de madeira de talhão de Eucalyptus sp. a partir dessas variáveis. O número total de árvores detectadas foi obtido com uso da filtragem de máxima local. A altura de plantas estimada pelo LiDAR apresentou tendência não significativa à subestimativa. A estimativa do DAP foi coerente com os valores encontrados no inventário florestal; porém, também mostrou tendência à subestimativa, em razão do comportamento observado quanto à altura. A variável número de fustes apresentou valores próximos aos observados nas parcelas do inventário. O LiDAR subestimou o volume total de madeira do talhão em $11,4 \%$, em comparação ao volume posto na fábrica. A tendência de subestimação da altura das árvores (em média, cerca de 5\%) impactou a estimativa do volume individual de árvores e, consequentemente, a do volume do talhão. No entanto, é possível gerar equações de regressão que estimam o DAP com boa precisão, a partir de dados de altura de plantas obtidos pelo LiDAR. O modelo parabólico é o que possibilita as melhores estimativas da produção volumétrica dos talhões de eucalipto.

Termos para indexação: Eucalyptus, inventário florestal, LiDAR, modelos biométricos, sensoriamento remoto.

\section{Determining timber volume of eucalyptus stands by airborne laser scanning}

\begin{abstract}
The objective of this work was to evaluate the possibility of estimating the diameter at breast height (DBH) with tree height and number data derived from airborne laser scanning (LiDAR, light detection and ranging) dataset, and to determine the timber volume of an Eucalyptus sp. stand from these variables. The total number of detected trees was obtained using a local maxima filtering. Plant height estimated by LiDAR showed a nonsignificant tendency to underestimation. The estimate for DBH was coherent with the results found in the forest inventory; however, it also showed a tendency towards underestimation due to the observed behavior for height. The variable number of stems showed values close to the ones observed in the inventory plots. LiDAR underestimated the total timber volume in the stand in $11.4 \%$, compared to the total volume delivered to the industry. The underestimation tendency of tree height (5\% mean value) impacted the individual tree volume estimate and, consequently, the stand volume estimate. However, it is possible to obtain regression equations that estimate DBH with good precision, from the LiDAR plant height derived data. The parabolic model is the one that provides the best estimates for timber volumetric yield of eucalyptus stands.
\end{abstract}

Index terms: Eucalyptus, forest inventory, LiDAR, biometric models, remote sensing.

\section{Introdução}

A crescente necessidade de aprimorar as estimativas de inventários florestais tem motivado estudos com dados de sensoriamento remoto, como auxílio à quantificação volumétrica de povoamentos florestais.

O uso do "light detection and ranging" (LiDAR), que é um escâner a laser aerotransportado, para a determinação da elevação de terrenos iniciou-se na segunda metade da década de 1970 (Lillesand \& Kiefer, 2000) e, desde os anos 1980, seu emprego para aplicações florestais tem avançado significativamente. A maior disponibilidade de imagens de alta resolução espacial torna viável a obtenção de informações de sensoriamento remoto relativas a árvores individuais, 
e não mais apenas para talhões ou povoamentos (Disperati et al., 2007).

Atualmente, o LiDAR tem uma gama de aplicações em diversas áreas, como arqueologia (Harmon et al., 2006), planejamento costeiro, avaliação de risco de inundações, telecomunicações, redes de transmissão de energia, florestas, agricultura, petróleo, transportes, planejamento urbano e mineração, entre outras (Giongo et al., 2010). No Brasil, no entanto, a linha de pesquisa com o equipamento ainda é incipiente, com destaque para os trabalhos de Castro \& Centeno (2005), Castro (2006), Zandoná et al. (2008), Macedo (2009), Rodriguez et al. (2010), Zonete (2009) e Zonete et al. (2010). No mundo, há vários estudos para estimar parâmetros de parcelas com o uso do LiDAR, para a diminuição da intensidade amostral. Esses estudos têm sido desenvolvidos com contagem de árvores individuais em campo, com a quantificação do estoque florestal e da biomassa, como nos trabalhos de Naesset \& Bjerknes (2001), Popescu et al. (2002, 2003), Maltamo et al. (2004), Naesset (2004), Yu et al. (2004), Tiede et al. (2005), Crow et al. (2007), Jupp et al. (2007), Naesset (2007), Heurich \& Thoma (2008), Lauri et al. (2008) Sasaki et al. (2008), e Ioki et al. (2010).

O desenvolvimento de novas técnicas, bem como a validação das já existentes para condições brasileiras, é importante para consolidar o uso desta tecnologia no setor florestal. Entre as variáveis que podem ser obtidas com dados do LiDAR estão altura, densidade de plantio, diâmetro de copa, distribuição espacial de plantas, índices de heterogeneidade dos talhões, ocorrências de falhas em reboleiras, entre outras. O volume de madeira do povoamento é a variável mais valiosa para a gestão florestal, tanto no âmbito estratégico como no operacional. A altura das árvores é uma variável dendrométrica de difícil aquisição no inventário florestal, mas apresenta forte correlação com o volume de madeira de um talhão e, portanto, é de grande importância para a determinação do incremento volumétrico em talhões homogêneos (Skovsgaard \& Vanclay, 2008).

Zandoná et al. (2008) utilizaram informações de altura derivadas de LiDAR para estimar o diâmetro de copa por meio de regressão linear. Posteriormente, uma segunda regressão, que utilizava o diâmetro de copa e a altura como parâmetros, foi utilizada pelos autores para estimar a variável dendrométrica diâmetro à altura do peito (DAP). Os autores então utilizaram os percentis $10,30,50,70$ e 90, para classificar as estimativas das variáveis biométricas, que foram utilizadas em modelos para estimar o volume de árvores individuais. Modelos de regressão permitem estimar o DAP a partir da altura das árvores. Esses modelos são tradicionalmente usados de forma inversa; assim, para a determinação da altura a partir do volume, Ribeiro et al. (2010) utilizaram o modelo de Curtis e obtiveram bons resultados na determinação da altura a partir do DAP.

O objetivo deste trabalho foi avaliar a possibilidade de se estimar o diâmetro à altura do peito (DAP), com os dados de altura e de número de árvores derivados do escâner a laser aerotransportado (LiDAR, "light detection and ranging"), e determinar o volume de talhão de Eucalyptus sp. a partir dessas variáveis.

\section{Material e Métodos}

A área experimental está situada no extremo sul da Bahia, entre as coordenadas $16^{\circ} 05^{\prime} 10^{\prime \prime} \mathrm{S}, 39^{\circ} 25^{\prime} 30^{\prime \prime} \mathrm{W}$ e $16^{\circ} 05^{\prime} 40 " \mathrm{~S}, 39^{\circ} 25^{\prime} 00^{\prime \prime} \mathrm{W}$ (Figura 1). O clima da região, conforme classificação de Köppen, é do tipo tropical equatorial, com chuvas distribuídas durante o ano inteiro e média anual de $1.200 \mathrm{~mm}$. A temperatura média é de $24^{\circ} \mathrm{C}$, com pequena amplitude, por se tratar de região costeira. A área compreende um talhão de Eucalyptus sp. de 23,5 ha, plantado no espaçamento de $5,00 \times 2,40 \mathrm{~m}$, com sete anos de idade à época do voo.

O talhão foi inventariado na mesma semana do voo com o LiDAR, tendo-se considerado uma suficiência amostral de quatro parcelas. O número de parcelas foi definido com vistas à obtenção de um erro no inventário inferior a $10 \%$, para a variável volume, e uma superfície de análise de pelo menos $2.000 \mathrm{~m}^{2}$. Assim, quatro parcelas circulares de $530,93 \mathrm{~m}^{2}$, com o centro georreferenciado com DGPS, foram distribuídas aleatoriamente pelo talhão, conforme os círculos em vermelho da Figura 1. Nessas parcelas, foi feita a medição do DAP, em todas as árvores, e da altura das seis primeiras árvores. Para a estimativa das alturas totais das demais árvores, utilizou-se a relação hipsométrica proposta por Campos et al. (1984), ajustada para o talhão em estudo, pela equação HTest $=\exp \left(\beta_{0}+\beta_{1} /\right.$ DAP $+\beta_{2}$ Ln Hdom $)+\varepsilon$, em que: HTest é a altura estimada; DAP é o diâmetro à altura do peito (1,30 $\mathrm{m}$ do solo); Hdom é a altura dominante; $\beta_{\mathrm{i}}$ representa os parâmetros estimados; e $\varepsilon$ é o erro. 
Um teste t pareado foi realizado para averiguar se havia diferenças significativas entre os valores médios de altura em cada parcela, obtidos pelo LiDAR e pelo inventário florestal.

A estimativa de volume foi realizada pelo modelo de Schumacher \& Hall por $\mathrm{V}_{\mathrm{i}}=\beta_{0} \mathrm{DAP}^{\beta 1} \mathrm{HT}^{\beta 2}+\varepsilon$, em que: $V_{\mathrm{i}}$ é o volume total estimado na iésima árvore; DAP é o diâmetro à altura do peito; HT é a altura total, $\beta_{\mathrm{i}}$ representa os parâmetros estimados; e $\varepsilon$ é o erro. A equação foi ajustada com informações de 65 árvores, cubadas rigorosamente para o clone existente no talhão, com a mesma idade e em diferentes classes diamétricas.

Os dados do LiDAR foram coletados com o sistema ALTM 3100, acoplado a um avião bimotor Piper Seneca II. O voo foi realizado à velocidade de $150 \mathrm{~km} \mathrm{~h}^{-1}$, à altura de $1.000 \mathrm{~m}$, diâmetro do feixe de $20 \mathrm{~cm}$, divergência do feixe de $0,3 \mathrm{mrad}$, ângulo de varredura de $15^{\circ}$, frequência de varredura de $58,7 \mathrm{~Hz}$ e intensidade de 1,5 ponto $\mathrm{m}^{-2}$. $\mathrm{O}$ erro planimétrico foi de $0,5 \mathrm{~m}(1,0 \mathrm{~s})$, o altimétrico de $0,15 \mathrm{~m}(1,0 \mathrm{~s})$, e a área de cobertura do laser foi de $0,32 \mathrm{~m}$. Os dados dos sinais do LiDAR referentes às áreas dos talhões foram organizados e calibrados com base nas informações de latitude, longitude e altitude $(\mathrm{x}, \mathrm{y}, \mathrm{z})$, pulsos $\left(1^{\circ}, 2^{\circ}\right.$ e $3^{\mathrm{o}}$ retornos) e intensidade do sinal. Esses fatores podem influenciar a densidade da nuvem de pontos resultante, que pode variar de 0,5 a 5 pontos $\mathrm{m}^{-2}$. A variação é decorrente do tipo de produto encomendado, da altura e da velocidade de voo, e de características do sensor laser. A precisão altimétrica do LiDAR é de aproximadamente $15 \mathrm{~cm}$, segundo Crow et al. (2007).

Após a estruturação das informações, analisou-se a sobreposição lateral de $30 \%$ entre as linhas de voo e a rota de voo da aeronave, para separar os pontos sobrepostos. As informações de sobreposição não foram consideradas no presente estudo. O ruído advindo da sobreposição pode ter sido causado pelo provável deslocamento do dossel pelo vento, nas diferentes passagens da aeronave, em que a interporlação pelo método IDW ("inverse distance weighted interpolation") são mais realçadas (Oliveira et al., 2012). Os dados foram processados conforme Oliveira et al. (2012), tendo-se analisado duas classes de elevação, segundo o modelo digital de superfície (MDS): na primeira classe, todas as informações referentes ao primeiro pulso acima de $10 \mathrm{~m}$ de altura; e, na segunda, apenas as informações referentes ao primeiro pulso acima de $1 \mathrm{~m}$.

A altura das árvores foi obtida por meio da diferença entre os dados fornecidos pelo modelo digital de terreno (MDT) e os do MDS. Para isso, utilizaram-se regras

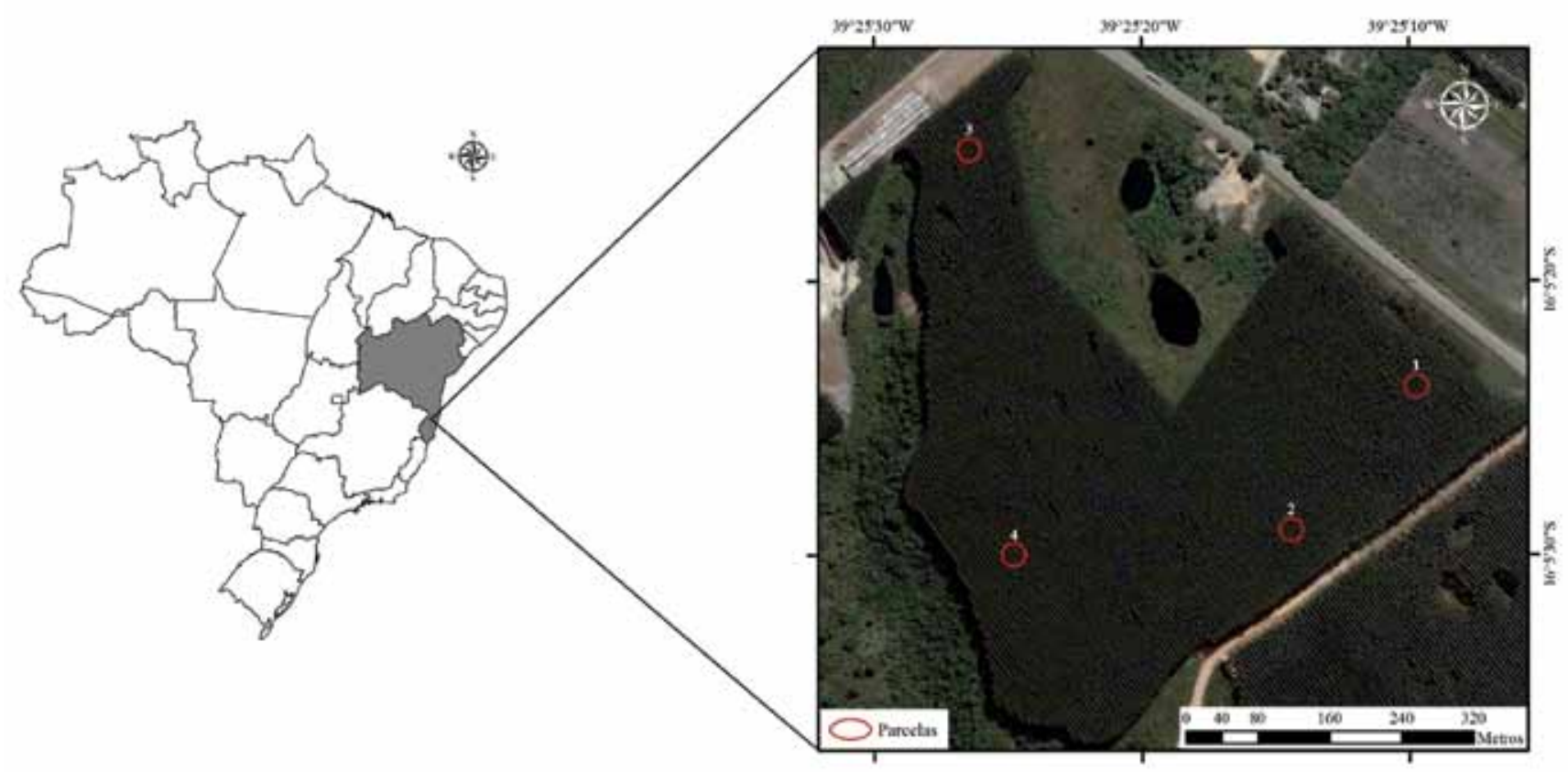

Figura 1. Localização geográfica do talhão escaneado com o LiDAR e parcelas circulares usadas no inventário florestal (em vermelho). 
de lógica binária, tendo-se avaliado as informações de pulso, altitude e angulação vertical e horizontal entre os pontos vizinhos, a partir do desvio-padrão das altitudes de cada pulso, do número de árvores e de suas respectivas alturas, obtidas pelo método proposto por Oliveira et al. (2012). De acordo com esse método, os dados brutos do LiDAR são organizados em função das informações $\mathrm{x}, \mathrm{y}$, $\mathrm{z}$ e da separação dos retornos $\left(1^{\circ}\right.$., $2^{\mathrm{o}}$. e $3^{\circ}$.), e classificados como solo, vegetação acima de $1 \mathrm{~m}$ de altura e vegetação acima de $10 \mathrm{~m}$, considerandose os dados de sobreposição do voo, que são retirados do processamento. A altura é calculada para todos os pontos, em relação ao MDT, e utilizada para geração do MDS considerando-se apenas o primeiro retorno; este modelo é rasterizado pelo método IDW. Este raster é submetido a um filtro de local máxima, com uso de janela móvel de $5 \times 5$, de onde são extraídos o número e a altura das árvores detectadas.

Para a determinação do volume, ajustou-se o modelo de regressão para estimação do DAP a partir da altura das árvores obtida pelo LiDAR. Para isso, foram testados quatro modelos tradicionais para a relação hipsométrica, tomados em sua forma inversa: modelo parabólico, DAP $=\beta_{0}+\beta_{1} \mathrm{HT}+\beta_{2} \mathrm{HT}^{2}+\varepsilon_{\mathrm{i}}$; modelo da linha reta, DAP $=\beta_{0}+\beta_{1} \mathrm{HT}+\varepsilon_{\mathrm{i}}$; modelo de Stofel, $\log (\mathrm{DAP})=\beta_{0}+\beta_{1} \log (\mathrm{HT})+\log \left(\varepsilon_{\mathrm{i}}\right) ;$ e modelo de Curtis, DAP $=\beta_{0}+\beta_{1} 1 / \log (\mathrm{HT})+\varepsilon_{\mathrm{i}}$.

Para a seleção do melhor modelo, avaliou-se o gráfico de resíduos, o erro-padrão da estimativa $(\%$ e $\mathrm{cm})$ e, por último, o coeficiente de determinação $\mathrm{R}^{2}$. Todos os modelos foram ajustados conforme os dados do inventário florestal, tendo-se utilizado a melhor equação para a estimativa do DAP, nas duas classes de dados do LiDAR (classe 1, primeiro pulso acima de $10 \mathrm{~m}$; e classe 2, primeiro pulso acima de $1 \mathrm{~m}$ ), interpolados pelo IDW por meio de uma janela móvel 5x5 (Oliveira et al., 2012).

Após a estimativa da altura, aplicou-se a equação ajustada para o modelo volumétrico de Schumacher \& Hall.

Os resultados das duas classes obtidas com LiDAR foram comparadas com as informações obtidas pelo inventário, para todo o talhão, com o registro da contagem do número de árvores por hectare, cortadas pelo harvester, e com o volume entregue na fábrica. Neste último caso, a madeira foi pesada e sua densidade foi estimada para a obtenção do volume; a medição ocorreu 35 dias após a colheita. A Figura 2 apresenta um resumo da sequência metodológica empregada neste estudo.

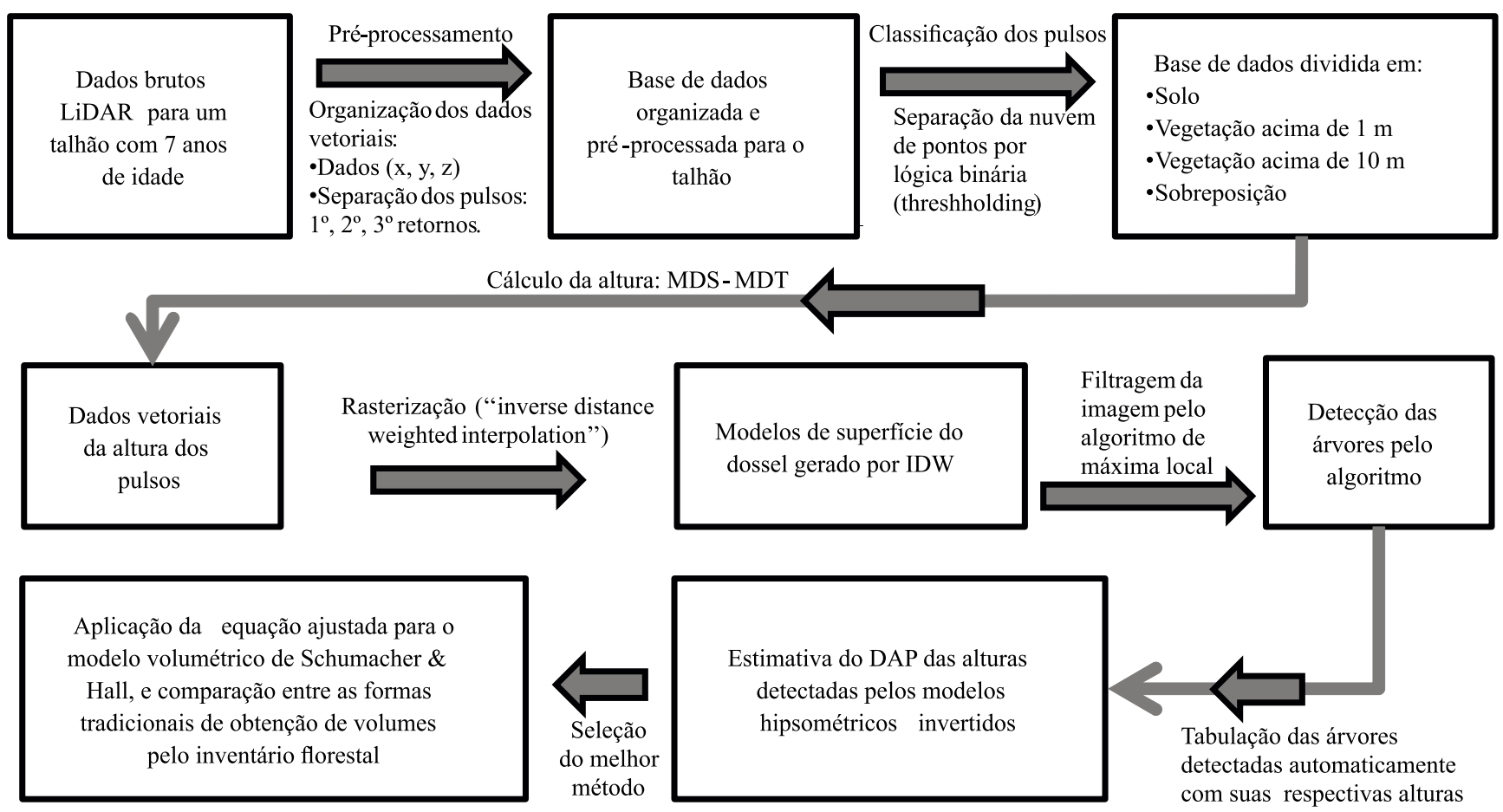

Figura 2. Fluxograma para a execução da metodologia proposta. 


\section{Resultados e Discussão}

As médias de altura obtidas pelo inventário florestal e pelo LiDAR, para a classe 1 (média $=1,65$, $\mathrm{DP}=1,738, \mathrm{~N}=4$ ) e para a classe 2 (média $=1,70$, $\mathrm{DP}=1,676, \mathrm{~N}=4$ ), não diferiram significativamente $[\mathrm{t}(3)=1,90$ e $\mathrm{p}$ bicaudal $=0,154$, para a classe 1 ; $\mathrm{e}$ $\mathrm{t}(3)=2,03$ e p bicaudal $=0,135$, para a classe 2)]. As Tabelas 1 e 2 apresentam uma síntese das informações obtidas no inventário florestal, realizado no talhão à época do voo. Os modelos de relação hipsométrica apresentaram boas estatísticas de ajuste, com alto coeficiente de determinação (média de 92\%) e baixos valores de erro (média do erro-padrão residual de $1,1 \mathrm{~cm}$ ). Estes valores estão de acordo com estudos com relação hipsométrica em eucalipto, tais como os de Soares et al. (2004) e Ribeiro et al. (2010).

Tabela 1. Síntese das informações obtidas pelo inventário de um talhão de Eucalyptus sp.

\begin{tabular}{lcccccc}
\hline Parcela & $\begin{array}{c}\mathrm{N}^{\text {o de }} \\
\text { fustes }\end{array}$ & DAP & $\begin{array}{c}\text { Altura } \\
\text { total }\end{array}$ & $\begin{array}{c}\text { Altura } \\
\text { dominante } \\
\text { (cm) }\end{array}$ & $\begin{array}{c}\text { Volume } \\
\text { individual }\end{array}$ & $\begin{array}{c}\text { Volume por } \\
\text { parcela }\end{array}$ \\
\hline 1 & 39 & 20,3 & 32,0 & 33,8 & 0,4696 & 18,31 \\
2 & 43 & 19,6 & 30,6 & 34,5 & 0,4473 & 19,23 \\
3 & 43 & 21,2 & 32,8 & 35,0 & 0,4549 & 19,55 \\
4 & 47 & 19,0 & 31,3 & 32,5 & 0,3668 & 17,24 \\
\hline
\end{tabular}

DAP, diâmetro à altura do peito.

Tabela 2. Síntese das medidas de precisão, obtidas pelo inventário, para a variável volume de madeira.

\begin{tabular}{lcc}
\hline Estatística & Estimativa & Unidade \\
\hline Variância & 1,08 & $\mathrm{~m}^{3} \mathrm{ha}^{-1}$ \\
Desvio-padrão & 1,04 & $\mathrm{~m}^{3} \mathrm{ha}^{-1}$ \\
Erro-padrão da média & 0,52 & $\mathrm{~m}^{3} \mathrm{ha}^{-1}$ \\
Erro-padrão da média & 2,79 & $\%$ \\
Volume médio & 18,59 & $\mathrm{~m}^{3} \mathrm{ha}^{-1}$ \\
Coeficiente de variação & 5,60 & $\%$ \\
Número de amostras & 4,00 & - \\
Erro no inventário & 1,65 & $\mathrm{~m}^{3}$ \\
Erro no inventário & 8,87 & $\%$ \\
\hline Intervalo de confiança & & \\
Parcela, limite inferior & 16,94 & $\mathrm{~m}^{3}$ \\
Parcela, limite superior & 20,23 & $\mathrm{~m}^{3}$ \\
Hectare, limite inferior & 318,97 & $\mathrm{~m}^{3}$ \\
Hectare, limite superior & 381,07 & $\mathrm{~m}^{3}$ \\
Talhão, limite inferior & $7.495,87$ & $\mathrm{~m}^{3}$ \\
Talhão, limite superior & $8.955,19$ & $\mathrm{~m}^{3}$ \\
\hline
\end{tabular}

Os dados de $\mathrm{R}^{2}$ e do erro-padrão residual apontaram para o modelo parabólico como o de melhor ajuste (Tabela 3). O gráfico de dispersão (Figura 3) também indica que houve melhor ajuste do modelo parabólico. Assim, o modelo foi selecionado para estimar o DAP das árvores a partir de seus dados de altura obtidos pelo LiDAR. Esse modelo, por ser essencialmente matemático, tem aplicação restrita ao intervalo de dados ao qual foi ajustado.

Os valores de DAP estimados apresentaram boa aderência aos valores obtidos no inventário florestal, nas parcelas 1 e 2 (Tabela 4), com diferença média de $-4 \%$. Já os valores de DAP para as parcelas 3 e 4 foram subestimados em 17,8\%. Esta subestimativa do DAP ocorreu em consequência da subestimativa da altura média pelo LiDAR, nas parcelas 3 e 4, que foi, em média, 9,8\% menor do que a altura obtida no inventário. Assim, se a variável altura for corretamente estimada pelo LiDAR, espera-se um bom acerto na variável DAP.

O modelo para estimar o volume de madeira, a partir do DAP $\left(\mathrm{V}=0,000041 \mathrm{DAP}^{1,76383} \times \mathrm{HT}^{1,165339}\right)$, apresentou os seguintes parâmetros: $\mathrm{R}^{2}$ ajustado, 98,40; erro-padrão residual $\left(\mathrm{m}^{3}\right), 0,020851$; e $\mathrm{CV}$, $5,3 \%$. Os dados do LiDAR na classe 2 foram os que apresentaram menor diferença quanto à estimativa da produção volumétrica, em comparação à produção medida na fábrica (Tabela 5). Todos os dados volumétricos para o talhão, excetuando-se os da classe 1, estiveram dentro do intervalo de confiança do inventário (Tabela 2); porém, a classe 1 excedeu o limite inferior em $256,28 \mathrm{~m}^{3}$ e gerou um erro de subestimativa de $14 \%$ em relação ao volume posto na fábrica.

O volume obtido com "harvester" foi um pouco mais alto do que o posto em fábrica (Tabela 5). Isso pode ocorrer, pois este equipamento, quando em operação,

Tabela 3. Parâmetros para o ajuste dos modelos analisados.

\begin{tabular}{lcccc}
\hline Modelo $^{(1)}$ & Equação & $\begin{array}{c}\mathrm{R}^{2} \\
\text { ajustado }\end{array}$ & $\begin{array}{c}\text { Erro-padrão } \\
\text { residual }(\mathrm{cm})\end{array}$ & $\begin{array}{c}\mathrm{CV} \\
(\%)\end{array}$ \\
\hline Parabólico & $\mathrm{DAP}=127,2-7,88 \mathrm{HT}+0,141 \mathrm{HT}^{2}$ & 87,0 & 0,82 & 4,10 \\
Linha reta & $\mathrm{DAP}=-15,7+1,118 \mathrm{HT}$ & 85,5 & 0,87 & 4,33 \\
Stofel & $\mathrm{Ln}(\mathrm{DAP})=-3,1179+1,7646 \mathrm{Ln}(\mathrm{HT})$ & 86,4 & 0,86 & 4,27 \\
Curtis & $\mathrm{DAP}=142,2-423 \times 1 / \mathrm{LN}(\mathrm{HT})$ & 84,5 & 0,90 & 4,47 \\
\hline
\end{tabular}

(1)Modelo parabólico, DAP $=\beta_{0}+\beta_{1} \mathrm{HT}+\beta_{2} \mathrm{HT}^{2}+\varepsilon_{\mathrm{i}}$; modelo da linha reta, $\mathrm{DAP}=\beta_{0}+\beta_{1} \mathrm{HT}+\varepsilon_{i}$; modelo de Stofel, $\log (\mathrm{DAP})=\beta_{0}+\beta_{1} \log (\mathrm{HT})+$ $\log \left(\varepsilon_{\mathrm{i}}\right)$; e modelo de Curtis, DAP $=\beta_{0}+\beta_{1} 1 / \log (\mathrm{HT})+\varepsilon_{\mathrm{i}}$. 
tende a contar árvores mais de uma vez, quando elas se quebram durante a operação. A tendência da contagem individual, de subestimar o número de árvores encontradas nas parcelas do inventário florestal, não era esperada e deve ter ocorrido em razão da supressão de árvores muito próximas na contagem individual.
A subestimativa para o volume estimado com os dados do LiDAR, em relação aos dados do inventário florestal, pode ter sido provocada pela subestimativa das alturas detectadas nos pontos de máxima. Assim, em razão da altura subestimada, o volume também foi subestimado em relação ao posto na fábrica. No entanto,
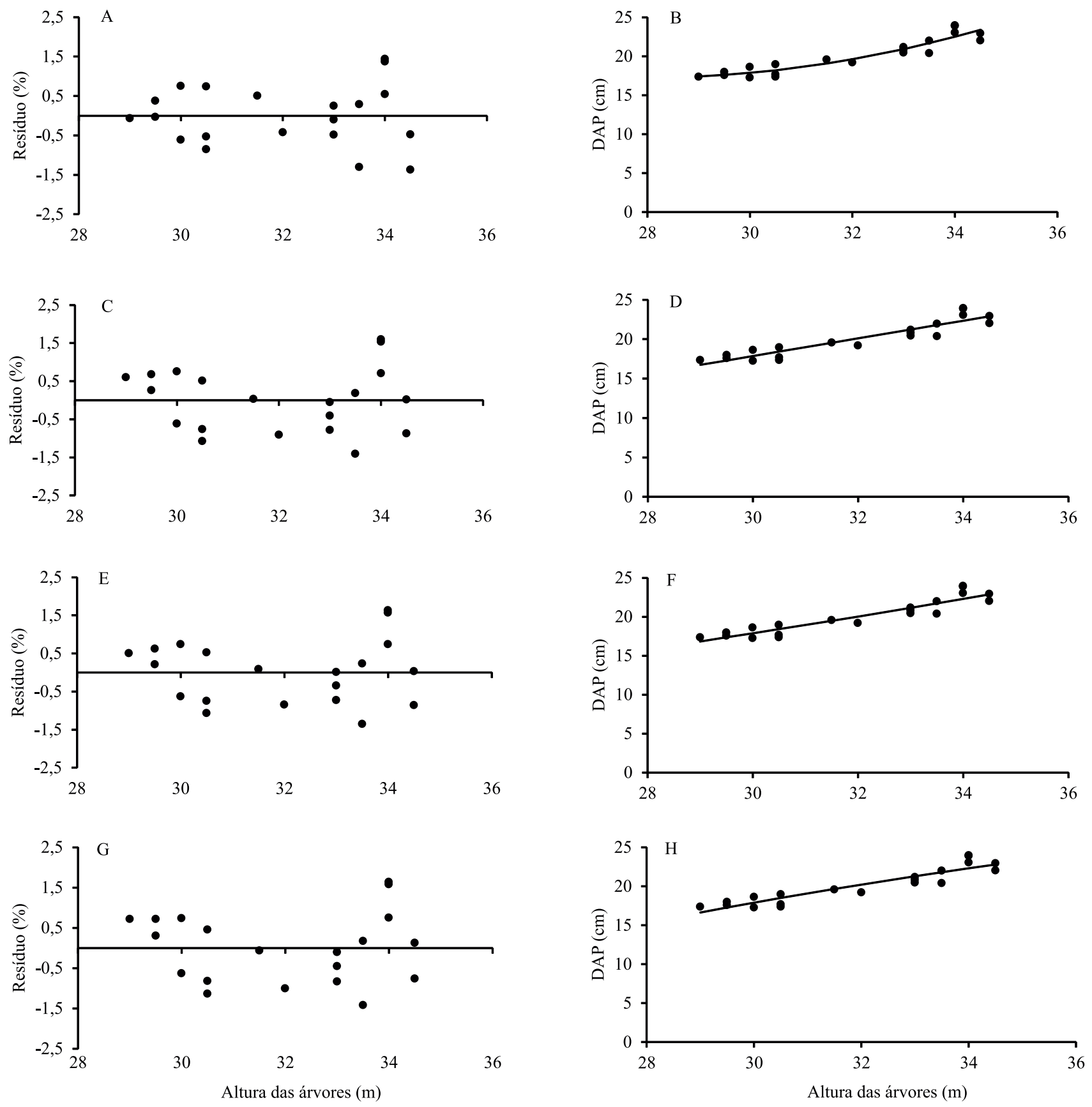

Figura 3. Dispersão dos resíduos e inclinação da reta para os modelos: A e B, parabólico; C e D, linha reta; E e F, Stofel; e $\mathrm{G}$ e H, Curtis. 
o volume total da classe 2 ficou dentro do intervalo de confiança do inventário. A altura é uma variável dendrométrica de grande importância para o inventário florestal (Skovsgaard \& Vanclay, 2008) que, se não tivesse sido subestimada pela contagem automática, teria proporcionado estimativas de volume mais próximas do real. A existência de sobreposição ou de árvores muito próximas foi responsável pela subestimativa do número de árvores captado pelo LiDAR, o que também foi relatado por Oliveira et al. (2012), e pelo fato de os pontos de máxima não terem captado os picos das árvores nas classes 1 e 2. Assim, é possível que o LiDAR acerte o

Tabela 4. Valores médios de altura, obtidos com o LiDAR, e DAP, estimado com o modelo parabólico, e valores médios de altura e DAP obtidos pelo inventário florestal.

\begin{tabular}{lccc}
\hline Parâmetro & Classe 1 & Classe 2 & Inventário \\
\hline & \multicolumn{3}{c}{ Parcela 1 } \\
Altura média $(\mathrm{m})$ & 31,94 & 31,81 & 32,50 \\
DAP $(\mathrm{cm})$ & 19,52 & 19,36 & 20,35 \\
\hline \multicolumn{3}{c}{ Parcela 2} \\
Altura média (m) & 30,79 & 30,70 \\
DAP $(\mathrm{cm})$ & 18,12 & 18,02 & 30,58 \\
\hline & \multicolumn{3}{c}{ Parcela 3 } \\
Altura média $(\mathrm{m})$ & 29,88 & 29,89 \\
DAP $(\mathrm{cm})$ & 17,16 & 17,15 \\
\hline & \multicolumn{3}{c}{ Parcela 4} \\
Altura média $(\mathrm{m})$ & 28,03 & 28,03 \\
DAP $(\mathrm{cm})$ & 15,33 & 15,33 \\
\hline & \multicolumn{3}{c}{ Valor médio } \\
Altura média $(\mathrm{m})$ & 30,16 & 30,11 & 31,33 \\
DAP $(\mathrm{cm})$ & 17,53 & 17,47 & 31,81 \\
\hline
\end{tabular}

Tabela 5. Número de árvores e volume, encontrados com contagem visual, LiDAR, inventário, inventário pré-corte e "harvester".

\begin{tabular}{|c|c|c|c|c|c|c|}
\hline \multirow[t]{2}{*}{ Parâmetro } & \multicolumn{4}{|c|}{ Parcela } & \multirow{2}{*}{$\begin{array}{l}\text { Valor } \\
\text { médio }\end{array}$} & \multirow{2}{*}{$\begin{array}{l}\text { Valor total } \\
\text { (talhão) }\end{array}$} \\
\hline & 1 & 2 & 3 & 4 & & \\
\hline $\mathrm{N}^{\mathrm{o}}$ de árvores - contagem visual & 39 & 43 & 41 & 46 & 42 & 18.090 \\
\hline No de árvores - LiDAR classe 1 & 41 & 31 & 45 & 42 & 40 & 18.337 \\
\hline $\mathrm{N}^{\circ}$ de árvores - LiDAR classe 2 & 46 & 32 & 41 & 47 & 42 & 19.700 \\
\hline $\mathrm{N}^{\circ}$ de árvores - inventário & 39 & 43 & 43 & 47 & 43 & 18.986 \\
\hline $\mathrm{N}^{\circ}$ de árvores - pré-corte & - & - & - & - & - & 19.308 \\
\hline No de árvores - "harvester" & - & - & - & - & - & 20.019 \\
\hline Volume de árvore classe $1\left(\mathrm{~m}^{3}\right)$ & 0,44 & 0,37 & 0,33 & 0,25 & 0,35 & $7.239,59$ \\
\hline Volume de árvore classe $2\left(\mathrm{~m}^{3}\right)$ & 0,43 & 0,37 & 0,33 & 0,25 & 0,35 & $7.684,99$ \\
\hline Volume de árvore inventário $\left(\mathrm{m}^{3}\right)$ & 0,47 & 0,45 & 0,45 & 0,36 & 0,43 & $8.225,53$ \\
\hline Volume no inventário pré-corte $\left(\mathrm{m}^{3}\right)$ & - & - & - & - & 0,43 & 8.288 \\
\hline Volume no "harvester" $\left(\mathrm{m}^{3}\right)$ & - & - & - & - & - & 8.593 \\
\hline Volume total na fábrica $\left(\mathrm{m}^{3}\right)$ & - & - & - & - & - & 8.418 \\
\hline
\end{tabular}

Pesq. agropec. bras., Brasília, v.49, n.9, p.692-699, set. 2014

DOI: 10.1590/S0100-204X2014000900005 número total das árvores no talhão, em razão de erros compensativos no decorrer da análise. Macedo (2009) verificou que, apesar de os algoritmos para estimativa volumétrica que utilizam dados LiDAR subestimarem a quantidade de árvores e a altura, e superestimarem o DAP, a estimativa volumétrica se mantém dentro de um intervalo aceitável, tanto em termos de intervalo de confiança quanto de erro amostral.

Em geral, o deslocamento da copa das árvores, em áreas limítrofes das parcelas, causado pelo vento nas diferentes passagens da aeronave, faz com que elas sejam suprimidas ou acrescentadas ao cômputo total de árvores em cada parcela, o que seria responsável por erros, ainda que pequenos. Esses erros são responsáveis pela diferença entre o valor das informações observadas no inventário, na contagem visual e na detecção automática. Apesar disso, os dados indicam boa precisão quanto ao volume total do talhão para a classe 2. Portanto, o LiDAR é uma ferramenta promissora para o setor florestal, embora ainda demande estudos para sua correta implementação.

\section{Conclusões}

1. É possível gerar equações de regressão para estimar o DAP com boa precisão a partir de dados de altura obtidos pelo LiDAR, e o modelo parabólico é o que possibilita as melhores estimativas.

2. A subestimativa da altura das árvores detectadas pelo LiDAR é a principal responsável pela diferença entre o volume de madeira estimado com o uso equipamento e o volume posto em fábrica.

\section{Agradecimentos}

À Coordenação de Aperfeiçoamento de Pessoal de Nível Superior (Capes) e à Fundação de Amparo à Pesquisa do Estado de Minas Gerais (Fapemig), pelas bolsas de estudo.

\section{Referências}

CAMPOS, J.C.C.; RIBEIRO, J.O.; PAULA NETO, F. Inventário florestal nacional, reflorestamento: Minas Gerais. Brasília: Instituto Brasileiro de Desenvolvimento Florestal, 1984. 126p.

CASTRO, F. da C. e. Segmentação de imagens geradas por laser scanning aerotransportado para delimitação de árvores individuais em áreas de reflorestamento de eucaliptos. 2006. 76p. Dissertação (Mestrado) - Universidade Tecnológica Federal do Paraná, Curitiba. 
CASTRO, F.C. e; CENTENO, T.M. Segmentação de imagens geradas por perfilamento a laser para delimitação de árvores individuais em uma área de reflorestamento de eucaliptos. In: SIMPÓSIO BRASILEIRO DE SENSORIAMENTO REMOTO, 12., 2005, Goiânia. Anais. Goiânia: INPE, 2005. p.737-744.

CROW, P.; BENHAM, S.; DEVEREUX, B.J.; AMABLE, G.S. Woodland vegetation and its implications for archaeological survey using LiDAR. Forestry, v.80, p.241-252, 2007. DOI: 10.1093/ forestry/cpm018.

DISPERATI, A.A.; SANTOS, J.R. dos; OLIVEIRA FILHO, P.C. de; NEEFF, T. Aplicação da técnica "filtragem de locais máximas" em fotografia aérea digital para a contagem de copas em reflorestamento de Pinus elliottii. Scientia Forestalis, n.76, p.45-55, 2007.

GIONGO, M.; KOEHLER, H.S.; MACHADO, S. do A.; KIRCHNER, F.F.; MACHETTI, M. LiDAR: princípios e aplicações florestais. Pesquisa Florestal Brasileira, v.30, p.231-244, 2010. DOI: $10.4336 / 2010 . p f b .30 .63 .231$.

HARMON, J.M.; LEONE, M.P.; PRINCE, S.D.; SNYDER, M. LiDAR for archaeological landscape analysis: a case study of two eighteenth-century Maryland plantation sites. American Antiquity, v.71, p.649-670, 2006. DOI: 10.2307/40035883.

HEURICH, M.; THOMA, F. Estimation of forestry stand parameters using laser scanning data in temperate, structurally rich natural European beech (Fagus sylvatica) and Norway spruce (Picea abies) forests. Forestry, v.81, p.645-661, 2008. DOI: 10.1093/forestry/cpn038.

IOKI, K.; JUNICHI, I.; SASAKI, T.; MORIMOTO, Y.; KITADA, K. Estimating stand volume in broad-leaved forest using discrete-return LiDAR: plot-based approach. Landscape and Ecological Engineering, v.6, p.29-36, 2010. DOI: 10.1007/ s11355-009-0077-4.

JUPP, D.; CULVENOR, D.; LOVELL, G.; NEWHAM, G.; STRAHLER, A.; WOODCOCK, C. Estimating forest LAI profiles and structural parameters using a ground-based laser called Echidna. Tree Physiology, v.29, p.171-181, 2007. DOI: 10.1093/ treephys/tpn022.

LAURI, K.; JUSSI, P.; JUKKA, M.; AKI, S.; MATTI, M.; PETTERI, P.; JYRKI, K. The use of airborne laser scanning to estimate sawlog volumes. Forestry, v.81, p.499-509, 2008. DOI: 10.1093/forestry/cpn018.

LILLESAND, T.M.; KIEFER, R.W. Remote sensing and image interpretation. Madison: John Wiley, 2000. 724p.

MACEDO, R. de C. Estimativa volumétrica de povoamento clonal de Eucalyptus sp. através de laser scanner aerotransportado. 2009. 143p. Dissertação (Mestrado) - Instituto Nacional de Pesquisas Espaciais, São José dos Campos.

MALTAMO, M.; EERIKÄINEN, K.; PITKÄNEN, J.; HYYPPÄ, J.; VEHMAS, M. Estimation of timber volume and stem density based on scanning laser altimetry and expected tree size distribution functions. Remote Sensing of Environment, v.90, p.319-330, 2004. DOI: 10.1016/j.rse.2004.01.006.

NAESSET, E. Airbone laser scanning as method in operational foresty inventory: status of accuracy assessments accomplished in Scandinavia. Scandinavian Journal of Forest Research, v.22, p.433-442, 2007. DOI: 10.1080/02827580701672147.

NAESSET, E. Practical large-scale forest stand inventory using a small-footprint airborne scanning laser. Scandinavian Journal of Forest Research, v.19, p.164-179, 2004. DOI: $10.1080 / 02827580310019257$.

NAESSET, E.; BJERKNES, K.O. Estimating tree heights and number of stems in young forest stands using airborne laser scanner data. Remote Sensing of Environment, v.78, p.328-340, 2001. DOI: 10.1016/S0034-4257(01)00228-0.

OLIVEIRA, L.T. de; CARVALHO, L.M.T. de; FERREIRA, M.Z.; OLIVEIRA, T.C. de A.; ACERBI JUNIOR, F.W. Application of LIDAR to forest inventory for tree count in stands of Eucalyptus sp. Cerne, v.18, p.75-184, 2012. DOI: 10.1590/ S0104-77602012000200001.

POPESCU, S.C.; WYNNE, R.H.; NELSON, R.F. Estimating plot-level tree heights with lidar: local filtering with a canopy-height based variable window size. Computers and Electronics in Agriculture, v.37, p.71-95, 2002. DOI: 10.1016/ S0168-1699(02)00121-7.

POPESCU, S.C.; WYNNE, R.H.; NELSON, R.F. Measuring individual tree crown diameter with LiDAR and assessing its influence on estimating forest volume and biomass. Canadian Journal of Remote Sensing, v.29, p.564-577, 2003. DOI: 10.5589/ m03-027.

RIBEIRO, A.; FERRAZ FILHO, A.C.; MELLO, J.M.; FERREIRA, M.Z.; LISBOA, P.M.M.; SCOLFORO, J.R.S. Estratégias e metodologias de ajuste de modelos hipsométricos em plantios de Eucalyptus sp. Cerne, v.16, p.22-31, 2010.

RODRIGUEZ, L.C.E.; POLIZEL, J.L.; FERRAZ, S.F.B.; ZONETE, M.F.; FERREIRA, M.Z. Inventário florestal com tecnologia laser aerotransportada de plantios de Eucalyptus spp. no Brasil. Ambiência, v.6, p.67-80, 2010.

SASAKI, T.; IMANISHI, J.; IOKI, K.; MORIMOTO, Y.; KITADA, K. Estimation of leaf area index and canopy openness in broad-leaved forest using airborne laser scanner in comparison with high-resolution near-infrared digital photography. Landscape and Ecological Engineering, v.2, p.47-55, 2008. DOI: 10.1007/ s11355-008-0041-8.

SKOVSGAARD, J.P.; VANCLAY, J.K. Forest site productivity: a review of the evolution of dendrometric concepts for even-aged stands. Forestry, v.81, p.13-31, 2008. DOI: 10.1093/forestry/ cpm041.

SOARES, T.S.; SCOLFORO, J.R.S.; FERREIRA, S.O.; MELLO, J.M. Uso de diferentes alternativas para viabilizar a relação hipsométrica no povoamento florestal. Revista Árvore, v.28, p.845-854, 2004. DOI: 10.1590/S0100-67622004000600009.

TIEDE, D.; HOCHLEITNER, G.; BLASCHKE, T. A full GIS-based workflow for tree identification and tree crown delineation using laser scanning. In: STILLA, U.; ROTTENSTEINER, F.; HINZ, S. (Ed.). 3D city models, road databases, and traffic monitoring: concepts, algorithms, and evaluation. Vienna: IAPRS, 2005. p.9-14. 
YU, X.W.; HYYPPÄ, J.; KAARTINEN, H.; MALTAMO, M. Automatic detection of harvested trees and determination of forest growth using airborne laser scanning. Remote Sensing of Environment, v.90, p.451-462, 2004. DOI: $10.1016 / \mathrm{j}$. rse.2004.02.001.

ZANDONÁ, D.F.; LINGNAU, C.; NAKAJIMA, N.Y. Varredura a laser aerotransportado para estimativa de variáveis dendrométricas. Scientia Forestalis, v.36, p.295-306, 2008.
ZONETE,M.F.Análise do uso da tecnologia laseraerotransportado para inventários florestais em plantios clonais de Eucalyptus sp. no sul da Bahia. 2009. 95p. Dissertação (Mestrado) - Escola Superior de Agricultura Luiz de Queiroz, Piracicaba.

ZONETE, M.F.; RODRIGUEZ, R.C.E.; PACKALÉN, P. Estimação de parâmetros biométricos de plantios clonais de eucalipto no sul da Bahia: uma aplicação da tecnologia laser aerotransportada. Scientia Forestalis, v.38, p.225-235, 2010.

Recebido em 7 de abril de 2014 e aprovado em 19 de agosto de 2014 